Gazi University
Journal of Science
$\mathrm{http} / /$ dergipark.gov.tr/gujs

\title{
Parameter Estimation Methods and Applications of the Power Topp-Leone Distribution
}

\author{
Mohamed ELGARHY ${ }^{1}$ (D) , Amal HASSAN ${ }^{2}$ (D) , Heba NAGY ${ }^{2, *}$ (i) \\ ${ }^{I}$ Al Mahalla Al Kubra, Algharbia - The Higher Institute of Commercial Sciences, Al Mahalla Al Kubra, 31951, Algharbia, Egypt \\ ${ }^{2} 5$ Dr. Ahmed Zoweil St., Dokki - Faculty of Graduate Studies for Statistical Research (Cairo University, Department of Mathematical Statistics), \\ 12613, Giza, Egypt
}

Highlights

-We propose a new generalization for Topp-Leone (PTL) distribution with one more shape parameter.

- PTL is argued through different estimation procedures.

- A comparison is implemented between different estimates through a simulation study.

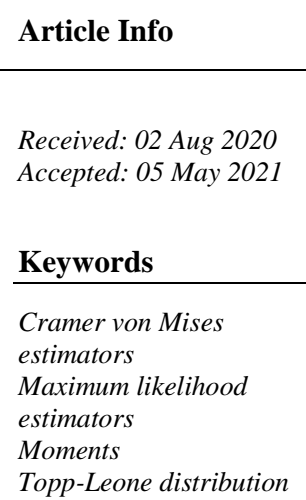

\begin{abstract}
We display the power Topp-Leone (PTL) distribution with two parameters. The following major features of the PTL distribution are investigated: quantile measurements, certain moment's measures, residual life function, and entropy measure. Maximum likelihood, least squares, Cramer von Mises, and weighted least squares approaches are used to estimate the PTL parameters. A numerical illustration is prepared to compare the behavior of the achieved estimates. Data analysis is provided to scrutinize the flexibility of the PTL model matched with Topp-Leone distribution.
\end{abstract}

\section{INTRODUCTION}

The origin of Topp-Leone (TL) distribution can be referred to [1] who introduced it. They obtained moments of the TL distribution as well as modeled it for some failure data. General formulae for some measures of moments and hazard rate function (hrf) motivation to the distribution have been discussed in [2]. [3] proposed the reflected generalized TL model, explored its properties and applied financial data in fitting income distribution. Some reliability measures of the TL model have been studied in [4]. Formulae of single and product moments based on order statistics were given in [5].

The density function of the TL distribution is J-shaped. A TL distribution's probability density function (pdf) is denoted by

$$
f(z ; \gamma)=2 z^{\gamma-1}(1-z)(2-z)^{\gamma-1}, \quad 0 \leq z \leq 1, \gamma>0 .
$$

The cumulative distribution function (cdf) related to Equation (1) is given by:

$$
F(z ; \gamma)=z^{\gamma}(2-z)^{\gamma}
$$

Constructions of the TL distribution have been found to be useful in several fields. Many efforts have been made by notable authors to propose new generalized and extended forms of TL model, for instance; TL inverse Weibull distribution [6]; TL geometric distribution [7], TL Nadarajah-Haghighi distribution [8], 
TL linear exponential distribution [9], new power TL (PTL) family [10], type II generalized TL family [11], type II PTL family [12], TL odd Fréchet distribution [13], inverse TL distribution [14] transmuted TL power function distribution [15], and TL inverse Lomax distribution [16] among others.

However, besides TL distribution there exist several unit distributions proposed recently by many researchers including; unit Johnson $S_{\mathrm{U}}$ distribution [17], unit generalized half normal distribution [18], logit slash distribution [19], truncated power Lomax distribution [20], and unit Burr-XII distribution [21]. So, the main aim here is to provide a new unit model related to the TL with an extra shape parameter, the so-called PTL distribution using the transformation, $T=Z^{1 / \beta}$, where $Z$ has TL distribution. The motivations of new distribution are (i) to give more desirable properties due to the extra parameter; (ii) to yield more flexibility in the form of its hrf and pdf, (iii) to produce kurtosis flexibility of the PTL distribution than the base TL model. In addition to real data analysis, properties and parameter estimates utilising maximum likelihood (ML), least squares (LS), weighted least squares (WLS), and Cramer-vonMises (CV) techniques are considered.

The following sections are included in the paper's layout. Section 2 defines the PTL distribution. Section 3 investigates the statistical characteristics of the PTL distribution. ML, LS, WLS, and CV estimators for model parameters are developed in Section 4. Section 5 includes a numerical inquiry. Section 6 contains data analysis. The essay concludes with a summary and conclusion.

\section{GENESIS OF POWER TOPP-LEONE DISTRIBUTION}

Herein, we contribute to the notion of PTL distribution.

\section{Definition:}

If we use the power transformation $T=Z^{1 / \beta}$, and $Z$ is a TL distribution with density function, we may say that a random variable $T$ has the power TL $(P T L)$ distribution. The cdf of the PTL distribution with shape parameters $\gamma$ and $\beta$, denoted by $T \sim P T L(\gamma, \beta)$, is as follows:

$$
F(t ; \gamma, \beta)=P(T \leq t)=P\left(Z^{1 / \beta} \leq t\right)=P\left(Z \leq t^{1 / \beta}\right)=F_{Z}\left(t^{1 / \beta}\right)
$$

where $F_{Z}\left(t^{1 / \beta}\right)$ is the cdf of TL distribution. Hence the cdf of the PTL distribution is written as:

$$
F(t ; \gamma, \beta)=t^{\gamma \beta}\left(2-t^{\beta}\right)^{\gamma} ; \quad 0 \leq t \leq 1, \quad \gamma, \beta>0 .
$$

The pdf of the PTL distribution corresponding to Equation (3) is formed as follows:

$$
f(t ; \gamma, \beta)=2 \gamma \beta t^{\gamma \beta-1}\left(2-t^{\beta}\right)^{\gamma-1}\left(1-t^{\beta}\right) ; \quad 0 \leq t \leq 1, \quad \gamma, \beta>0 .
$$

For, $\beta=1$, the pdf in Equation (4) yields the TL distribution. Plots of density for particular parameter values are exhibited in Figure 1. It is pdf is a very flexible model with diverse shapes. 

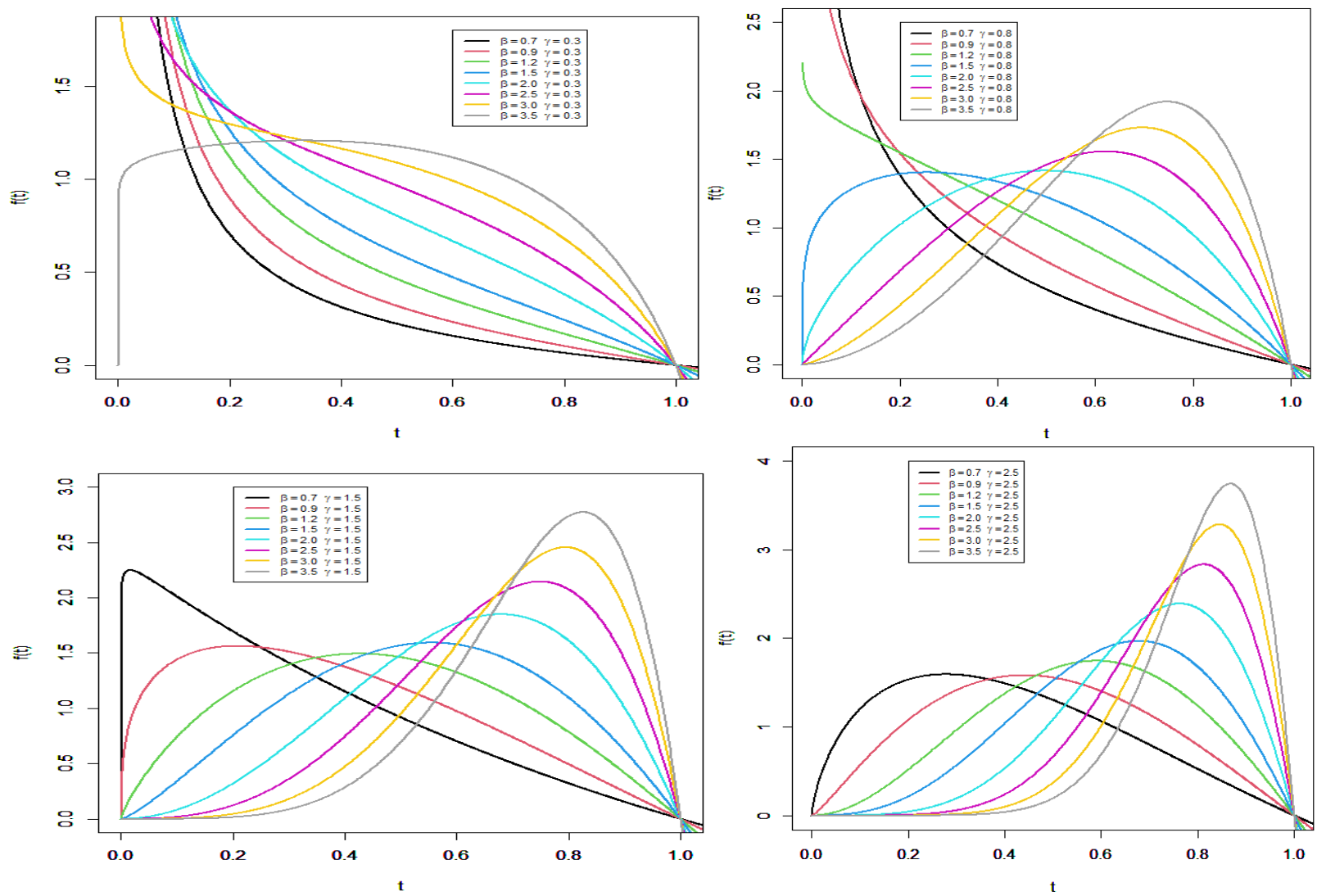

Figure 1. The PTL model's pdf plots for various parameter values

The survival function $S(t ; \gamma, \beta)$, the hrf $\zeta(t ; \gamma, \beta)$ and the cumulative hrf $v(t ; \gamma, \beta)$ of the PTL distribution are respectively, given by:

$$
\begin{aligned}
& S(t ; \gamma, \beta)=1-t^{\gamma \beta}\left(2-t^{\beta}\right)^{\gamma}, \\
& \zeta(t ; \gamma, \beta)=2 \gamma \beta t^{\gamma \beta-1}\left(2-t^{\beta}\right)^{\gamma-1}\left(1-t^{\beta}\right)\left\{1-t^{\gamma \beta}\left(2-t^{\beta}\right)^{\gamma}\right\}^{-1}, \\
& \text { and, } \\
& v(t ; \gamma, \beta)=-\ln \left[1-t^{\gamma \beta}\left(2-t^{\beta}\right)^{\gamma}\right] .
\end{aligned}
$$

Plots of the hrf are demonstrated for specific parameters value in Figure 2. We conclude that the hrf earns various shapes so it can be modeled for different real data.
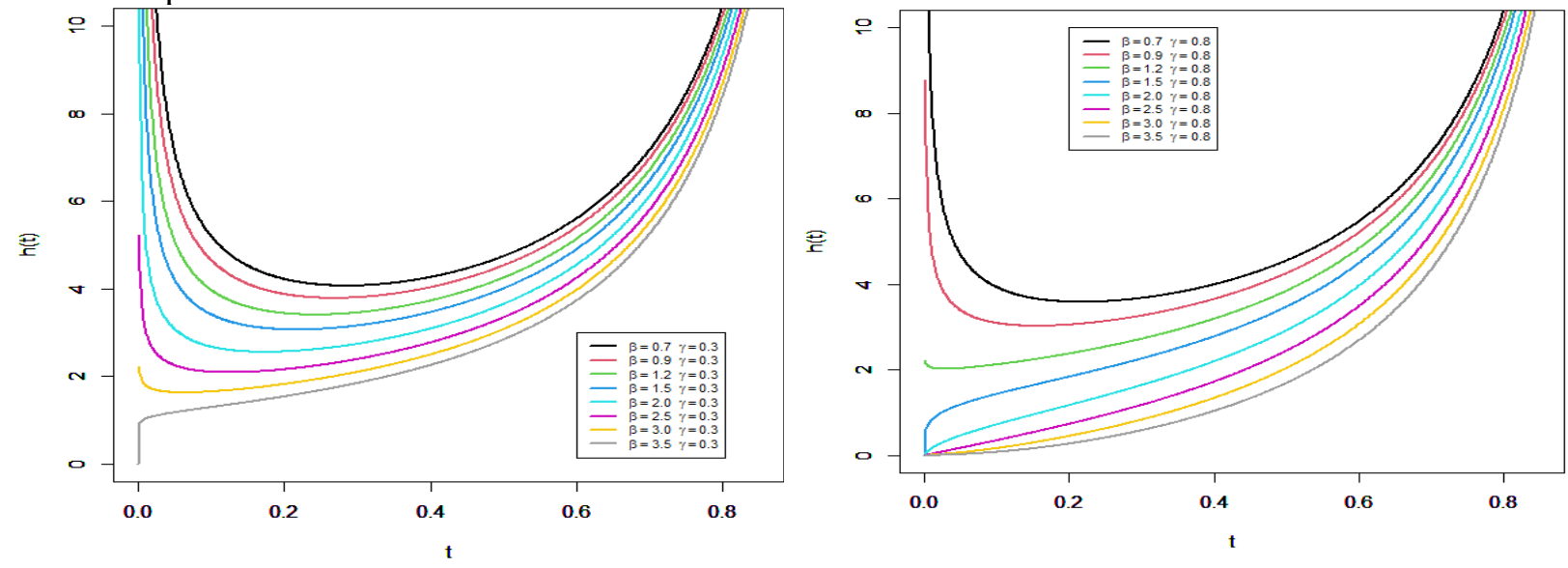

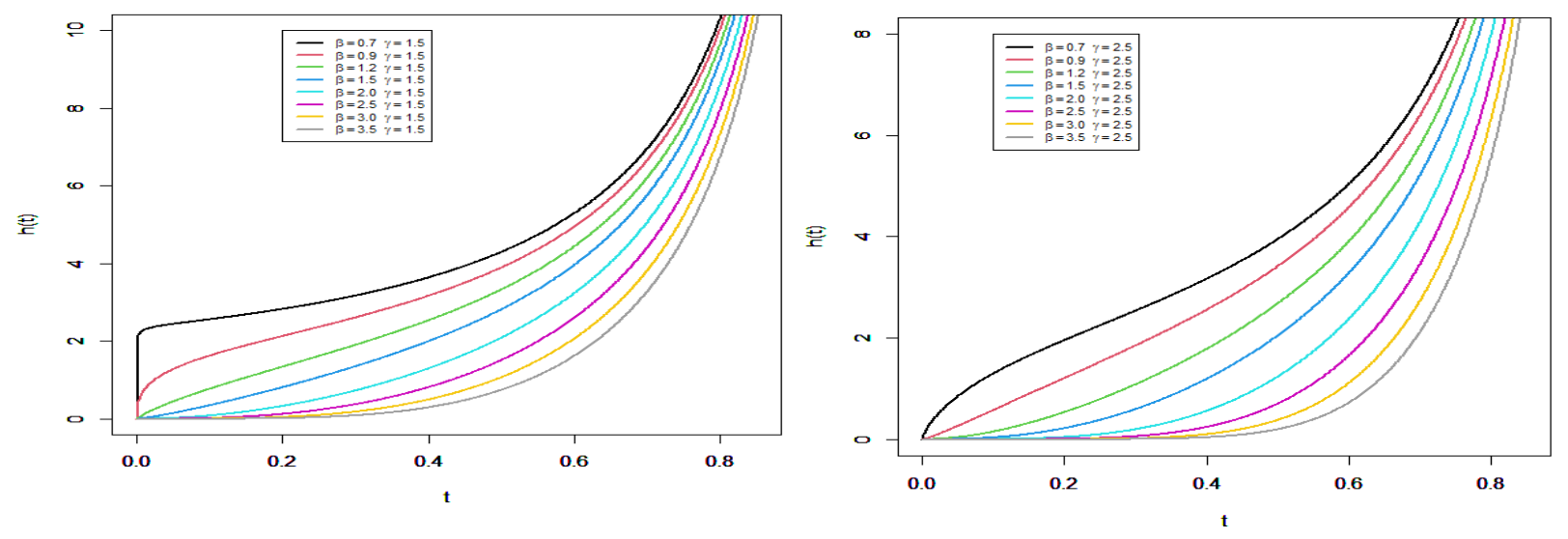

Figure 2. The PTL model's hrf plots for various parameter values

\section{SOME OF ESSENTIAL PROPERTIES}

Herein, the pivotal characteristics of the PTL distribution are formulated.

\subsection{Moments}

An explicit expression of the $r^{\text {th }}$ moment for the PTL is produced from (4) as follows

$$
\mu_{r}^{\prime}=2 \gamma \beta \int_{0}^{1} t^{r+\gamma \beta-1}\left(2-t^{\beta}\right)^{\gamma-1}\left(1-t^{\beta}\right) d t
$$

Suppose that, $y=t^{\beta}$, then Equation (5) will be as follows

$$
\mu_{r}^{\prime}=2 \gamma \int_{0}^{1} y(r / \beta)+\gamma-1(2-y)^{\gamma-1}(1-y) d y .
$$

Let, $z=1-y$, and by using the binomial expansion, then Equation (6) will be simplified to

$$
\begin{aligned}
\mu_{r}^{\prime} & =2 \gamma \sum_{q=0}^{\infty}\left(\begin{array}{c}
\gamma-1 \\
q
\end{array}\right) \int_{0}^{1}(1-z)^{(r / \beta)+\gamma-1} z^{q+1} d z \\
& =2 \gamma \sum_{q=0}^{\infty}\left(\begin{array}{c}
\gamma-1 \\
q
\end{array}\right) \mathrm{B}(r / \beta+\gamma, q+2) .
\end{aligned}
$$

Setting $r=1,2,3,4$ in Equation (7), we obtain the first four moments about zero. Furthermore, based on (7), the moment generating function of the PTL distribution is provided by:

$$
M_{t}(x)=\sum_{r=0}^{\infty} \frac{x^{r}}{r !} \mu_{r}^{\prime}=\sum_{r, q=0}^{\infty} \frac{2 \gamma x^{r}}{r !}\left(\begin{array}{c}
\gamma-1 \\
q
\end{array}\right) \mathrm{B}\left(\frac{r}{\beta}+\gamma, q+2\right) \text {. }
$$

The $r^{\text {th }}$ central moment $\left(\mu_{r}\right)$ of $T$ is given by:

$$
\mu_{r}=E\left(T-\mu_{1}^{\prime}\right)^{r}=\sum_{\ell=0}^{r}(-1)^{\ell}\left(\begin{array}{l}
r \\
\ell
\end{array}\right)\left(\mu_{1}^{\prime}\right)^{\ell} \mu_{r-\ell}^{\prime} .
$$

Moments values of order $1-4$, variance $\left(\sigma^{2}\right)$, skewness $(\mathrm{Sw})$ and kurtosis $(\mathrm{Kr})$ of the PTL distribution for specific parameters values (a) $(\gamma=0.3, \beta=0.8)$, (b) $(\gamma=0.3, \beta=1.5),(\mathrm{c})(\gamma=0.3, \beta=2.5)$, (d) $(\gamma=1.5, \beta=0.8),(e)(\gamma=1.5, \beta=1.5)$, (f) $(\gamma=1.5, \beta=2.5)$, and $(g)(\gamma=2.5, \beta=0.8)$, are recorded (Table 1). 
Table 1. Some Moments Measures for Assigned Parameters Values

\begin{tabular}{|cccccccc|}
\hline$\mu_{s}^{\prime}$ & $(\mathbf{a})$ & $(\mathbf{b})$ & $(\mathbf{c})$ & $(\mathbf{d})$ & $(\mathbf{e})$ & $(\mathbf{f})$ & $(\mathbf{g})$ \\
\hline$\mu_{1}^{\prime}$ & 0.113 & 0.223 & 0.346 & 0.346 & 0.53 & 0.668 & 0.442 \\
\hline$\mu_{2}^{\prime}$ & 0.045 & 0.104 & 0.186 & 0.173 & 0.328 & 0.477 & 0.244 \\
\hline$\mu_{3}^{\prime}$ & 0.024 & 0.061 & 0.118 & 0.103 & 0.222 & 0.358 & 0.154 \\
\hline$\mu_{4}^{\prime}$ & 0.015 & 0.041 & 0.083 & 0.068 & 0.16 & 0.278 & 0.105 \\
\hline$\sigma^{2}$ & 0.032 & 0.054 & 0.066 & 0.053 & 0.046 & 0.031 & 0.049 \\
\hline $\mathbf{S w}$ & 2.092 & 1.094 & 0.485 & 0.531 & -0.088 & -0.481 & 0.216 \\
\hline $\mathbf{K r}$ & 7.088 & 3.272 & 2.163 & 2.4 & 2.206 & 2.669 & 2.216 \\
\hline
\end{tabular}

It is reported from Table 1 that the values of $\mu_{1}^{\prime}$ and $\sigma^{2}$ get larger for increasing values of $\gamma$ and $\beta$. The behavior of distribution is left-skewed, right-skewed, and leptokurtic.

Next, $s^{\text {th }}$ incomplete moment of $T$ represented by $\pi_{s}(t)=E\left(X^{s} \mid X<t\right)$ is derived from Equation (4) as follows:

$$
\pi_{s}(t)=(2)^{\gamma} \gamma \beta\left\{\int_{0}^{t} x^{s+\gamma \beta-1}\left(1-x^{\beta} / 2\right)^{\gamma-1} d x-\int_{0}^{t} x^{s+\beta+\gamma \beta-1}\left(1-x^{\beta} / 2\right)^{\gamma-1} d x\right\} .
$$

Let, $y=x^{\beta} / 2$, then Equation (8) will be formed as follows:

$$
\begin{aligned}
& \pi_{s}(t)=2^{2 \gamma+\frac{s}{\beta}} \gamma \int_{0}^{(t)^{\beta}} y^{\frac{s}{\beta}+\gamma-1}(1-y)^{\gamma-1} d y-2^{2 \gamma+\frac{s}{\beta}+1} \gamma \int_{0}^{(t)^{\beta} / 2} y^{\frac{s}{\beta}+\gamma}(1-y)^{\gamma-1} d y \\
& =2^{2 \gamma+\frac{s}{\beta}} \gamma\left[\mathrm{B}\left(\frac{s}{\beta}+\gamma, \gamma, \frac{(t)^{\beta}}{2}\right)-2 \mathrm{~B}\left(\frac{s}{\beta}+\gamma+1, \gamma, \frac{(t)^{\beta}}{2}\right)\right],
\end{aligned}
$$

where $B(., ., t)$ is the incomplete beta function. The Bonferroni and Lorenz curves are two notable applications of incomplete moments. The PTL model's Lorenz and Bonferroni curves are given by:

$$
L Z(t)=\frac{2^{2 \gamma+\beta^{-1}}\left[\mathrm{~B}\left(\beta^{-1}+\gamma, \gamma,(t)^{\beta} / 2\right)-2 \mathrm{~B}\left(\gamma+\beta^{-1}+1, \gamma,(t)^{\beta} / 2\right)\right]}{\sum_{q=0}^{\infty}\left(\begin{array}{c}
\gamma-1 \\
q
\end{array}\right) \mathrm{B}\left(\beta^{-1}+\gamma, q+2\right)},
$$

and,

$$
B F(t)=\left[t^{\gamma \beta}\left(2-t^{\beta}\right)^{\gamma}\right]^{-1} L Z(t) .
$$

\subsection{Residual and Reversed Residual Life Functions}

The $n^{\text {th }}$ moment of the residual lifetime (MRL) is specified as follows:

$$
S_{n}(t)=(S(t))^{-1} \int_{t}^{\infty}(x-t)^{n} f(x) d x .
$$

the $n^{\text {th }}$ MRL of PTL model is given by applying binomial expansion and pdf (Equation 4) in Equation (10),

$$
\varsigma_{n}(t)=\frac{2 \gamma \beta}{S(t ; \gamma, \beta)} \sum_{r=0}^{n}(-1)^{n-r}\left(\begin{array}{l}
n \\
r
\end{array}\right) t^{n-r} \int_{t}^{1} x^{r+\gamma \beta-1}\left(2-x^{\beta}\right)^{\gamma-1}\left(1-x^{\beta}\right) d x .
$$

Let, $y=x^{\beta}$, then Equation (11) is as follows: 
$S_{n}(t)=\frac{2 \gamma}{S(t ; \gamma, \beta)} \sum_{r=0}^{n}(-1)^{n-r}\left(\begin{array}{l}n \\ r\end{array}\right) t^{n-r} \int_{t^{\beta}}^{1} y^{\frac{r}{\beta}+\gamma-1}(2-y)^{\gamma-1}(1-y) d y$.

After simplification, the $n^{\text {th }}$ MRL of the PTL distribution holds the form as follows:

$$
S_{n}(t)=\frac{2 \gamma}{S(t ; \gamma, \beta)} \sum_{r=0}^{n} \sum_{p=0}^{\infty}(-1)^{n-r}\left(\begin{array}{c}
\gamma-1 \\
p
\end{array}\right)\left(\begin{array}{l}
n \\
r
\end{array}\right) t^{n-r} \mathrm{~B}\left(\frac{r}{\beta}+\gamma, p+2,\left(1-t^{\beta}\right)\right),
$$

where, $\mathrm{B}(., ., x)$ denotes the incomplete beta function. When we put $n=1$ in (12), we get the mean residual life of the PTL distribution, which is important in a number of disciplines such as industrial dependability and biological science, among others.

Furthermore, the $n^{\text {th }}$ moment of reversed residual life (RRL) may be calculated as follows:

$\varepsilon_{n}(t)=\frac{1}{F(t)} \int_{0}^{t}(x-t)^{n} f(x) d x$.

Using binomial series and density (Equation 4), the $n^{\text {th }}$ moment of the RRL of the PTL distribution is generated as

$$
\varepsilon_{n}(t) \frac{1}{F(t ; \gamma, \beta)} \sum_{r=0}^{n}(-1)^{n-r}\left(\begin{array}{l}
n \\
r
\end{array}\right) t^{n-r} 2^{2 \gamma+n / \beta} \gamma\left[\mathrm{B}\left(n / \beta+\gamma, \gamma, t^{\beta} / 2\right)-2 \mathrm{~B}\left(n / \beta+\gamma+1, \gamma, t^{\beta} / 2\right)\right] .
$$

The mean of RRL is given by setting $n=1$ in Equation (13).

\subsection{Rényi Entropy}

Measure of uncertainty of $T$ is called the entropy. The Rényi entropy of $T$ for continuous random variable with range $R$ is given by:

$$
\rho_{R}(T)=\{1 /(1-\lambda)\} \ln \left\{\int_{R} f(t)^{\lambda} d t\right\}, \lambda \neq 1, \lambda>0 .
$$

The pdf; $(f(t ; \gamma, \beta))^{\lambda}$, of the PTL distribution can be formed as follows:

$$
\{f(t ; \gamma, \beta)\}^{\lambda}=(2 \gamma \beta)^{\lambda} t^{\lambda(\gamma \beta-1)}\left[2-t^{\beta}\right]^{\lambda(\gamma-1)}\left[1-t^{\beta}\right]^{\lambda} .
$$

Substituting Equation (15) in Equation (14) and let, $y=t^{\beta}$, then Equation (14) will be as follows:

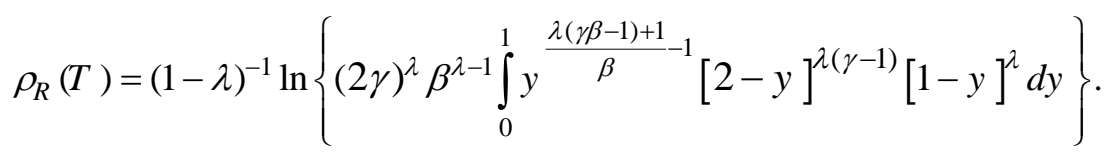

Using, $z=1-y$, and binomial expansion, then the Rényi entropy of the PTL is

$$
\rho_{R}(T)=(1-\lambda)^{-1} \ln \left\{(2 \gamma)^{\lambda} \beta^{\lambda-1} \sum_{j=0}^{\infty}\left(\begin{array}{c}
(\gamma-1) \lambda \\
j
\end{array}\right) \mathrm{B}\left(\frac{(\gamma \beta-1) \lambda+1}{\beta}, j+\lambda+1\right)\right\} .
$$

\subsection{Quantile Measures}

The quantile function of the PTL distribution, say $Q(p)=F^{-1}(p)$, of $T$ is produced by inverting Equation (3) 
as below:

$Q(p)=\left\langle 1-\left(1-p^{1 / \gamma}\right)^{0.5}\right\rangle^{1 / \beta}$,

where $p$ is a uniform random variable defined on $(0,1)$. The Bowley skewness (BO) and Moors kurtosis (MO), based on quantiles, are given by

$\mathrm{BO}=[Q(0.75)-2 Q(0.5)+Q(0.25)][Q(0.75)-Q(0.25)]^{-1}$,

and,

$\mathrm{MO}=Q(7 / 8)-Q(5 / 8)-Q(3 / 8)+Q(1 / 8)[Q(6 / 8)-Q(2 / 8)]^{-1}$,

where $Q($.) denotes the quantile function. The $\mathrm{BO}$ and $\mathrm{MO}$ plots for specific values of $\beta$ as function of $\gamma$ are represented in Figure 3.
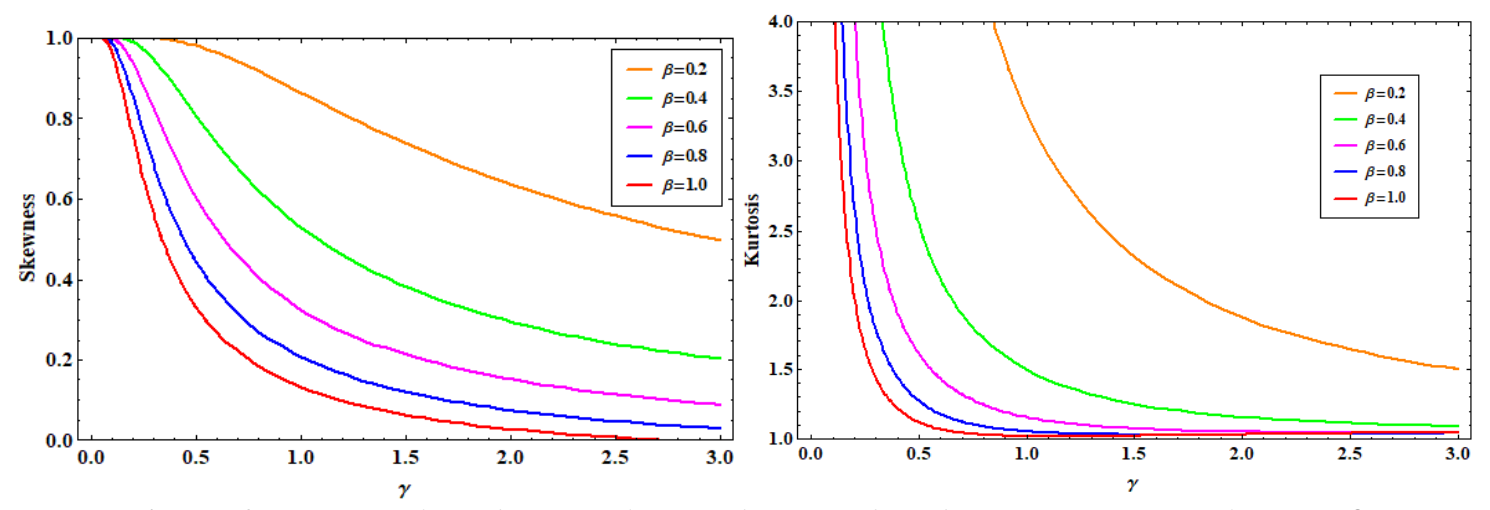

Figure 3. (a) BO Plots (b) MO plots of the PTL distribution for some values of $\beta$

\subsection{Stochastic Ordering}

Let $T$ and $Z$ are independent random variables with cdfs $F_{T}$ and $F_{Z}$ respectively, $T$ is said to be less than $Z$ if the following conditions are met:

- Stochastic order $\left(T \leq_{s r} Z\right)$, if $F_{T}(t) . t$ for all $) t\left({ }_{z} F \geq\right.$

- Likelihood ratio order (T.t is decreasing in $f_{T}(t) / f_{Z}(t)$ if $), Z \leq_{l r}$

- Hazard rate order $\left(T \leq_{h r} Z\right)$, if $h_{T}(t) . t$ for all $) t(z h \geq$

- Mean residual life order (T.t for all $) t(z m \geq) t\left(\right.$ тmif ), $Z \leq_{m r l}$

(see [22]).

Theorem 1: Let $T \sim \operatorname{PTL}\left(\gamma_{1}, \beta_{1}\right)$ and $Z \sim \operatorname{PTL}\left(\gamma_{2}, \beta_{2}\right)$. If $\beta_{1}<\beta_{2}$ and $\gamma_{1}=\gamma_{2}=\gamma$, then $T \leq_{l r} Z, T Z$, $\leq_{h r}$ $T \leq_{m r l} Z$, and $T . Z \leq_{s r}$.

\section{Proof.}

It is adequate to verify $f_{T}(t) / f_{Z}(t)$ is a non-increasing function of $t$; the likelihood ratio is

$\frac{f_{T}(t)}{f_{Z}(t)}=\frac{\beta_{1} t^{\gamma\left(\beta_{1}-\beta_{2}\right)}\left(2-t^{\beta_{1}}\right)^{\gamma-1}\left(1-t^{\beta_{1}}\right)}{\beta_{2}\left(2-t^{\beta_{2}}\right)^{\gamma-1}\left(1-t^{\beta_{2}}\right)}$. 
Therefore,

$$
\frac{d}{d t} \log \frac{f_{T}(t)}{f_{Z}(t)}=\frac{\gamma \beta_{1}-\gamma \beta_{2}}{t}-(\gamma-1)\left[\frac{\beta_{1} t^{\beta_{1}-1}}{2-t^{\beta_{1}}}+\frac{\beta_{2} t^{\beta_{2}-1}}{2-t^{\beta_{2}}}\right]-\left[\frac{\beta_{1} t^{\beta_{1}-1}}{1-t^{\beta_{1}}}-\frac{\beta_{2} t^{\beta_{2}-1}}{1-t^{\beta_{2}}}\right]<0 .
$$

Thus, $f_{T}(t) / f_{Z}(t)$ is a non-increasing in $t$ and hence $T \leq_{l r}$ Z. Likewise, we conclude for $T \leq_{h r} Z, T \leq_{m r l}$ $Z$, and $T . Z \leq_{s r}$.

\subsection{Stress-Strength Model}

Here, the stress-strength reliability parameter, denoted by $R$, is obtained. Let $T_{1} \sim \operatorname{PTL}\left(\gamma_{1}, \beta\right)$ and $T_{2} \sim \operatorname{PTL}$ $\left(\gamma_{2}, \beta\right)$ are independent. The reliability parameter $R$, of the PTL distribution is obtained as follows:

$$
R=2 \gamma_{1} \beta \int_{0}^{1} t^{\gamma_{1} \beta+\gamma_{2} \beta-1}\left(2-t^{\beta}\right)^{\gamma_{1}+\gamma_{2}-1}\left(1-t^{\beta}\right) d t
$$

Let $\mathrm{Z}=\left(1-t^{\beta}\right)$, then Equation (17) is as follows:

$$
\begin{aligned}
R & =2 \gamma_{1} \int_{0}^{1} z(1-z)^{\gamma_{1}+\gamma_{2}-1}(1+z)^{\gamma_{1}+\gamma_{2}-1} d z \\
& =2 \gamma_{1} \sum_{j=0}^{\infty}\left(\begin{array}{c}
\gamma_{1}+\gamma_{2}-1 \\
j
\end{array}\right) \mathrm{B}\left(\gamma_{1}+\gamma_{2}, j+2\right) .
\end{aligned}
$$

\subsection{Order Statistics}

Suppose $T_{1}, T_{2}, \ldots, T_{n}$ is a random sample that has cdf (3) and pdf (4). Let $T_{(1)}, T_{(2)}, \ldots, T_{(n)}$ be the associated order statistics (OS). The pdf of the $m^{\text {th }}$ OS is defined as:

$$
f_{T_{(m)}}(t)=\frac{n !}{(m-1) !(n-m) !}\{F(t)\}^{m-1}\{1-F(t)\}^{n-m} f(t)=\sum_{p=0}^{n-m} \Upsilon_{p}\{F(t)\}^{m+p-1} f(t)
$$

where $\Upsilon_{m}=\frac{n !}{(m-1) !(n-m) !}(-1)^{p}\left(\begin{array}{c}n-m \\ p\end{array}\right)$. It follows from Equations (3) and (4) that

$$
\begin{aligned}
f_{T_{(m)}}(t) & =2 \gamma \beta \sum_{p=0}^{n-m} t^{\gamma \beta[m+p]-1}\left[2-t^{\beta}\right]^{\gamma[m+p]-1}\left[1-t^{\beta}\right] \\
& =\sum_{p=0}^{n-m} W_{p, m} f(t ; \gamma(m+p), \beta),
\end{aligned}
$$

where, $0<t<1, W_{p, m}=\Upsilon_{m}(m+p)^{-1}$, and $f(t ; \gamma(m+p), \beta)$ is the pdf of the PTL $\sim(\gamma(m+p), \beta)$. Specifically, the pdf of first and largest OS are obtained for $m=1$ and $m=n$ respectively.

\section{ESTIMATION OF PARAMETERS}

Here, we deal with the estimation for the PTL parameters using four frequentist estimation methods.

\subsection{Estimators}

Let $T_{1}, T_{2}, \ldots, T_{n}$ is a complete random sample from the PTL distribution. The log-likelihood function of the PTL distribution, say $\ln L$, for the population parameters is given by: 
$\ln L=n \ln (2 \gamma \beta)+\sum_{i=1}^{n}(\gamma \beta-1) \ln t_{i}+(\gamma-1) \sum_{i=1}^{n} \ln \left[2-t_{i}{ }^{\beta}\right]+\sum_{i=1}^{n} \ln \left[1-t_{i}{ }^{\beta}\right]$.

The partial derivatives of $\ln L$ for $\gamma$ and $\beta$ are:

$$
\begin{aligned}
& \frac{\partial \ln L}{\partial \gamma}=(n / \gamma)+\left(\sum_{i=1}^{n} \beta \ln t_{i}\right)+\sum_{i=1}^{n}\left\langle\ln \left(2-t_{i}{ }^{\beta}\right)\right\rangle, \\
& \frac{\partial \ln L}{\partial \beta}=(n / \beta)+\gamma \sum_{i=1}^{n} \ln t_{i}-\sum_{i=1}^{n}\left(t_{i}{ }^{\beta}\right) \ln t_{i}\left[\frac{\gamma\left(1-t_{i}{ }^{\beta}\right)+1}{\left(2-t_{i}{ }^{\beta}\right)\left(1-t_{i}{ }^{\beta}\right)}\right] .
\end{aligned}
$$

Solving the non-linear equations $\partial \ln L / \partial \gamma=0$ and $\partial \ln L / \partial \beta=0$ numerically, so we get the ML estimators of parameters, i.e. $\hat{\gamma}$ and $\hat{\beta}$.

\subsection{LS and WLS Estimators}

The LS technique is suggested for estimating distribution parameters by minimizing the sum of square errors between actual and anticipated data. That is to say;

$$
\sum_{i=1}^{n}\left[\left[F\left(t_{(i)}\right)\right]-E\left(F\left(t_{(i)}\right)\right)\right]^{2} \text {. }
$$

The WLS estimator of the population parameters ([23]), is yielded by minimizing the following

$$
\sum_{i=1}^{n} \frac{(n+1)^{2}(n+2)}{i(n-i+1)}\left[F\left(t_{(i)}\right)-E\left(F\left(t_{(i)}\right)\right)\right]^{2}
$$

concerning the unknown parameters. Assume that $T_{(1)}, T_{(2)}, \ldots, T_{(n)}$, are the ordered observations of the PTL associated with a random sample of size $n, T_{1}, T_{2}, \ldots, T_{n}$. As a result, the LS and WLS estimators of $\gamma$ and $\beta$ say, $\tilde{\gamma}, \tilde{\beta}$ and $\bar{\gamma}, \bar{\beta}$ are obtained, respectively, by minimizing the following

$$
\sum_{i=1}^{n}\left\langle t_{(i)}^{\gamma \beta}\left(2-t_{(i)}^{\beta}\right)^{\gamma}-(i /(n+1))\right\rangle^{2}
$$

and,

$$
\sum_{i=1}^{n}\left(\frac{(n+1)^{2}(n+2)}{i(n-i+1)}\right)\left\langle t_{(i)}^{\gamma \beta}\left(2-t_{(i)}^{\beta}\right)^{\gamma}-(i /(n+1))\right\rangle^{2},
$$

owing to $\gamma$ and $\beta$.

\subsection{Estimators}

This approach is classified as a sort of minimal distance estimator since it is based on the difference between the cdf estimate and the empirical distribution function. [24] said that the estimator's bias is lower than that of other minimal distance estimators. The aforementioned estimators are created by reducing

$$
\mathrm{CV}(\gamma, \beta)=\frac{1}{12 n}+\sum_{i=1}^{n}\left\{\left[t_{(i)}^{\gamma \beta}\left(2-t_{(i)}^{\beta}\right)^{\gamma}\right]-\frac{2 i-1}{2 n}\right\}^{2},
$$

owing to unkown parameters.

\section{SIMULATION STUDY}

To illustrate the behaviour of different estimates, a numerical demonstration is presented. The PTL distribution generates 1000 random samples of size $n=50,100$ and 200. The parameter values are as 
follows, $\mathrm{i}=(\gamma=0.5, \beta=1), \mathrm{ii}=(\gamma=1, \beta=1), \mathrm{iii}=(\gamma=1.5, \beta=1), \mathrm{iv}=(\gamma=0.5, \beta=2), \mathrm{v}=(\gamma=1, \beta=2)$, and $\mathrm{vi}=(\gamma=1.5, \beta=2)$. The ML estimate (MLE), LS estimate (LSE), WLS estimate (WLSE) and CV estimate (CVE) of $\gamma$ and $\beta$ are computed. Then, all estimates and their mean squared errors (MSEs) are seated in Tables 2-7.

Table 2. Different Estimates and their MSEs at $(\gamma=0.5, \beta=1)$

\begin{tabular}{|cccccccccc|}
\hline $\boldsymbol{n}$ & Parameter & MLE & MSE & LSE & MSE & WLSE & MSE & CVE & MSE \\
\hline \multirow{2}{*}{$\mathbf{5 0}$} & $\gamma$ & 0.711 & 0.454 & 0.559 & 0.289 & 0.584 & 0.301 & 0.669 & $\mathbf{0 . 4 9 5}$ \\
\cline { 2 - 10 } & $\beta$ & 1.119 & 0.487 & 1.319 & 0.829 & 1.214 & 0.513 & 1.161 & $\mathbf{0 . 5 6 4}$ \\
\hline \multirow{2}{*}{$\mathbf{1 0 0}$} & $\gamma$ & 0.56 & 0.096 & 0.509 & 0.086 & 0.512 & 0.059 & 0.546 & $\mathbf{0 . 2 9 2}$ \\
\cline { 2 - 9 } & $\beta$ & 1.112 & 0.229 & 1.183 & 0.296 & 1.123 & 0.17 & 1.11 & $\mathbf{0 . 2 3 6}$ \\
\hline \multirow{2}{*}{$\mathbf{2 0 0}$} & $\gamma$ & 0.523 & 0.039 & 0.488 & 0.026 & 0.492 & 0.0 & 0.507 & $\mathbf{0 . 2 7}$ \\
\cline { 2 - 9 } & $\beta$ & 1.068 & 0.11 & 1.114 & 0.122 & 1.091 & 0.091 & 1.079 & $\mathbf{0 . 1 0 9}$ \\
\hline
\end{tabular}

Table 3. Different Estimates and their MSEs at $(\gamma=1, \beta=1)$

\begin{tabular}{|cccccccccc|}
\hline $\boldsymbol{n}$ & Parameter & MLE & MSE & LSE & MSE & WLSE & MSE & CVE & MSE \\
\hline \multirow{2}{*}{$\mathbf{5 0}$} & $\gamma$ & 1.275 & 1.27 & 0.862 & 0.599 & 0.934 & 0.681 & 1.054 & $\mathbf{1 . 1 9 1}$ \\
\cline { 2 - 11 } & $\beta$ & 1.222 & 0.598 & 1.524 & 0.959 & 1.424 & 0.738 & 1.391 & $\mathbf{0 . 7 7 9}$ \\
\hline \multirow{2}{*}{$\mathbf{1 0 0}$} & $\gamma$ & 1.055 & 0.373 & 0.816 & 0.263 & 0.833 & 0.197 & 0.869 & $\mathbf{0 . 3 3 1}$ \\
\cline { 2 - 10 } & $\beta$ & 1.143 & 0.198 & 1.338 & 0.32 & 1.285 & 0.273 & 1.308 & $\mathbf{0 . 3 0 7}$ \\
\hline \multirow{2}{*}{$\mathbf{2 0 0}$} & $\gamma$ & 0.965 & 0.152 & 0.721 & 0.144 & 0.811 & 0.071 & 0.749 & $\mathbf{0 . 1 4 6}$ \\
\cline { 2 - 10 } & $\beta$ & 1.121 & 0.106 & 1.344 & 0.211 & 1.206 & 0.11 & 1.309 & $\mathbf{0 . 1 8 3}$ \\
\hline
\end{tabular}

Table 4. Different Estimates and their MSEs at $(\gamma=1.5, \beta=1)$

\begin{tabular}{|cccccccccc|}
\hline \multirow{n}{*}{$\boldsymbol{N}$} & Parameter & MLE & MSE & LSE & MSE & WLSE & MSE & CVE & MSE \\
\hline \multirow{2}{*}{$\mathbf{5 0}$} & $\gamma$ & 1.495 & 1.184 & 1.143 & 1.067 & 1.243 & 1.114 & 1.422 & $\mathbf{1 . 7 3 4}$ \\
\cline { 2 - 10 } & $\beta$ & 1.344 & 0.594 & 1.601 & 1.076 & 1.479 & 0.738 & 1.423 & $\mathbf{0 . 7 6 3}$ \\
\hline \multirow{2}{*}{$\mathbf{1 0 0}$} & $\gamma$ & 1.29 & 0.482 & 1.037 & 0.517 & 1.101 & 0.433 & 1.158 & $\mathbf{0 . 5 1 6}$ \\
\cline { 2 - 9 } & $\beta$ & 1.271 & 0.253 & 1.472 & 0.484 & 1.385 & 0.346 & 1.377 & $\mathbf{0 . 3 5 7}$ \\
\hline \multirow{2}{*}{$\mathbf{2 0 0}$} & $\gamma$ & 1.183 & 0.272 & 1.01 & 0.373 & 1.078 & 0.275 & 1.036 & $\mathbf{0 . 1 5 9}$ \\
\cline { 2 - 9 } & $\beta$ & 1.255 & 0.145 & 1.387 & 0.239 & 1.307 & 0.164 & 1.368 & $\mathbf{0 . 2 2 8}$ \\
\hline
\end{tabular}

Table 5. Different Estimates and their MSEs at $(\gamma=0.5, \beta=2)$

\begin{tabular}{|cccccccccc|}
\hline \multirow{n}{n}{} & Parameter & MLE & MSE & LSE & MSE & WLSE & MSE & CVE & MSE \\
\hline \multirow{2}{*}{$\mathbf{5 0}$} & $\gamma$ & 0.759 & 0.414 & 0.615 & 0.257 & 0.65 & 0.284 & 0.712 & $\mathbf{1 . 9 9 8}$ \\
\cline { 2 - 10 } & $\beta$ & 2.006 & 1.144 & 2.417 & 2.791 & 2.135 & 1.399 & 2.124 & $\mathbf{1 . 8 2 7}$ \\
\hline \multirow{2}{*}{$\mathbf{1 0 0}$} & $\gamma$ & 0.618 & 0.126 & 0.558 & 0.079 & 0.597 & 0.087 & 0.613 & $\mathbf{2 . 0 1}$ \\
\cline { 2 - 9 } & $\beta$ & 2.012 & 0.577 & 2.167 & 0.913 & 2.015 & 0.533 & 1.968 & $\mathbf{0 . 5 4 1}$ \\
\hline \multirow{2}{*}{$\mathbf{2 0 0}$} & $\gamma$ & 0.573 & 0.052 & 0.551 & 0.038 & 0.559 & 0.049 & 0.565 & $\mathbf{2 . 0 9 6}$ \\
\cline { 2 - 9 } & $\beta$ & 1.964 & 0.286 & 2.009 & 0.279 & 2.032 & 0.348 & 1.98 & $\mathbf{0 . 2 9 8}$ \\
\hline
\end{tabular}

Table 6. Different Estimates and their MSEs at $(\gamma=1, \beta=2)$

\begin{tabular}{|cccccccccc|}
\hline $\boldsymbol{n}$ & Parameter & MLE & MSE & LSE & MSE & WLSE & MSE & CVE & MSE \\
\hline \multirow{2}{*}{50} & $\gamma$ & 1.333 & 0.994 & 1.019 & 0.842 & 1.036 & 0.557 & 1.195 & $\mathbf{1 . 5 1 2}$ \\
\cline { 2 - 10 } & $\beta$ & 2.15 & 1.185 & 2.62 & 2.599 & 2.455 & 1.688 & 2.335 & $\mathbf{1 . 9 2 5}$ \\
\hline \multirow{2}{*}{$\mathbf{1 0 0}$} & $\gamma$ & 1.11 & 0.363 & 0.947 & 0.179 & 0.968 & 0.172 & 1.02 & $\mathbf{1 . 2 0 3}$ \\
\cline { 2 - 9 } & $\beta$ & 2.177 & 0.673 & 2.349 & 0.889 & 2.281 & 0.615 & 2.226 & $\mathbf{0 . 6 6 2}$
\end{tabular}




\begin{tabular}{|llllllllll|}
\hline \multirow{2}{*}{200} & $\gamma$ & 1.047 & 0.153 & 0.929 & 0.071 & 0.971 & 0.09 & 0.963 & $\mathbf{1 . 1 4 1}$ \\
\cline { 2 - 16 } & 2.096 & 0.308 & 2.21 & 0.299 & 2.193 & 0.375 & 2.168 & $\mathbf{0 . 2 9 1}$ \\
\hline
\end{tabular}

Table 7. Different Estimates and their MSEs at $(\gamma=1.5, \beta=2)$

\begin{tabular}{|cccccccccc|}
\hline $\boldsymbol{n}$ & Parameter & MLE & MSE & LSE & MSE & WLSE & MSE & CVE & MSE \\
\hline \multirow{2}{*}{$\mathbf{5 0}$} & $\gamma$ & 1.753 & 1.701 & 1.314 & 1.054 & 1.372 & 1.38 & 1.527 & $\mathbf{2 . 0 7 3}$ \\
\cline { 2 - 9 } & $\beta$ & 2.331 & 1.599 & 2.849 & 3.139 & 2.701 & 2.023 & 2.612 & $\mathbf{2 . 3 2 6}$ \\
\hline \multirow{2}{*}{$\mathbf{1 0 0}$} & $\gamma$ & 1.527 & 0.464 & 1.216 & 0.541 & 1.156 & 0.371 & 1.277 & $\mathbf{0 . 8 7 9}$ \\
\cline { 2 - 9 } & $\beta$ & 2.243 & 0.683 & 2.665 & 1.455 & 2.61 & 0.919 & 2.508 & $\mathbf{1 . 0 1 7}$ \\
\hline \multirow{2}{*}{$\mathbf{2 0 0}$} & $\gamma$ & 1.476 & 0.225 & 1.206 & 0.211 & 1.124 & 0.276 & 1.219 & $\mathbf{0 . 7 8 1}$ \\
\cline { 2 - 9 } & $\beta$ & 2.183 & 0.394 & 2.445 & 0.507 & 2.542 & 0.554 & 2.444 & $\mathbf{0 . 5 4 2}$ \\
\hline
\end{tabular}

From Tables $2-7$ we reach to the following:

- The MSE of $\gamma$ and $\beta$ for all estimates decrease as $n$ increases.

- Table 2 shows that $\bar{\gamma}$ gets the smallest MSE while CVE of $\gamma$ takes the most MSE. Also, the WLSE has the least MSE of $\beta$ while LSE has the most MSE at $\gamma=0.5$ and $\beta=1$.

- The MSE of $\hat{\gamma}$ decreases as $\gamma$ decreases for all values of $\gamma$ and $\beta$ (see Figure 4 and Table 4).

- The MSE of $\tilde{\beta}$ decreases as $\beta$ decreases for all values of $\gamma$ and $\beta$ (see Figure 5 and Table 5).

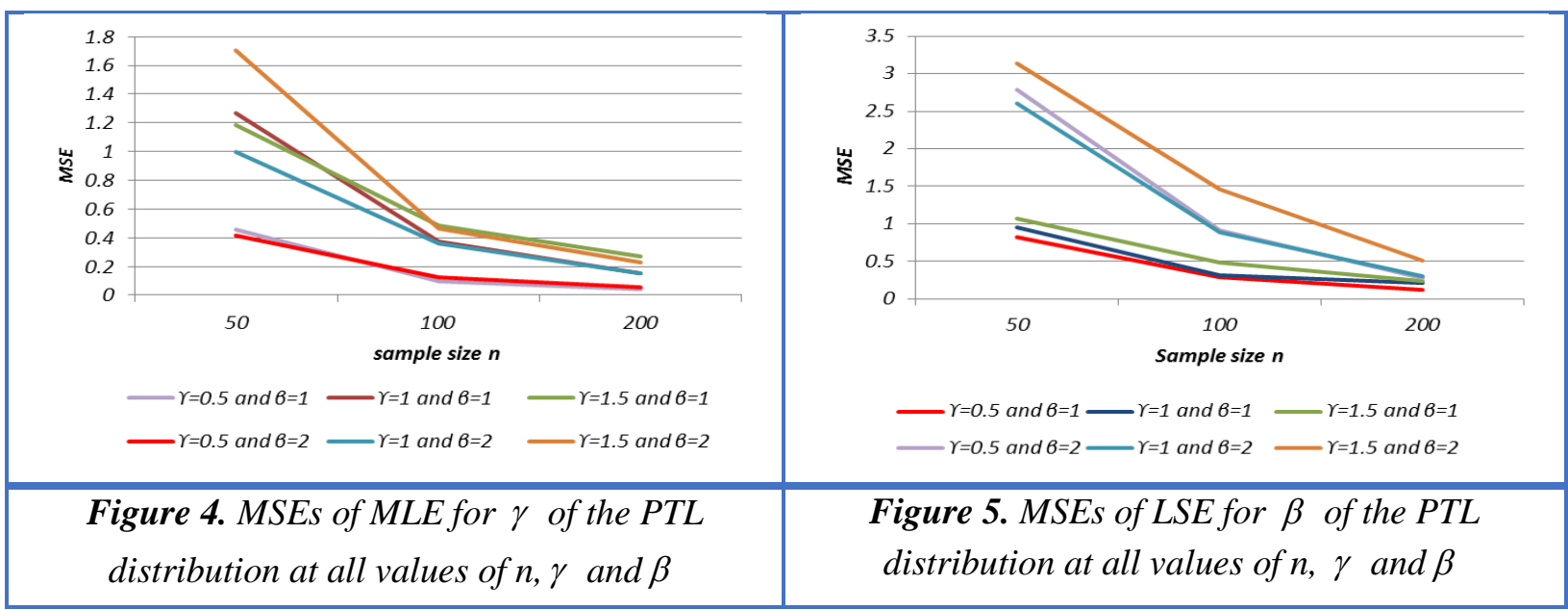

- The MSEs of the MLE, LSE, WLSE and CVE of $\beta$ get the largest values for all $n$ at $\gamma=1.5$ and $\beta=2$.

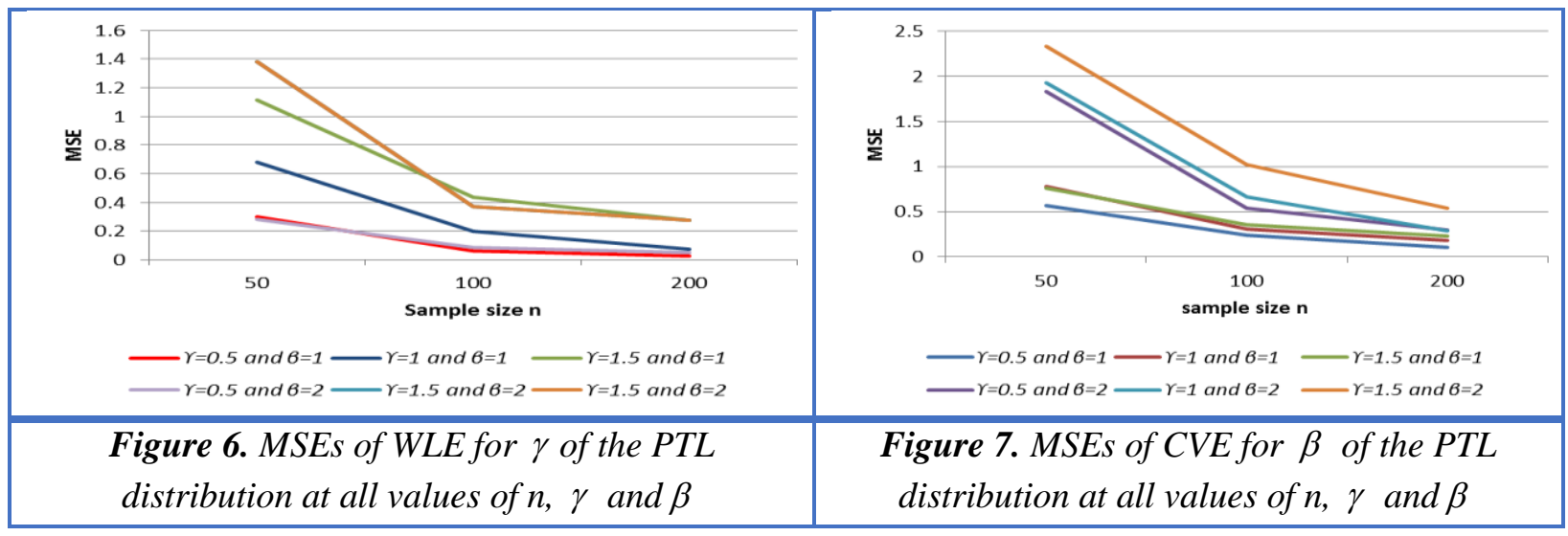


- The MSEs of the MLE, LSE, WLSE and CVE of $\gamma$ get the smallest values for all $n$ at $\gamma=0.5$ and $\beta=1$.

- The MSEs of the MLE, LSE, WLSE and CVE of $\gamma$ get the largest values for all $n$ at $\gamma=1.5$ and $\beta=2$. While, the MSEs of the MLE, LSE, WLSE and CVE of $\beta$ get the smallest values for all $n$ at $\gamma=0.5$ and $\beta=1$.

- The MSE of the WLE for $\gamma$ decreases as $\gamma$ decreases for all values of $\gamma$ and $\beta$ (see Figure 6).

- The MSE of the LSE for $\beta$ decreases as $\beta$ decreases for all values of $\gamma$ and $\beta$ (see Figure 7).

\section{REAL DATA ANALYSIS}

PTL model goodness-of-fit compared to several one-parameter rivals such as TL, Kumaraswamy (Ku) distribution, beta (B) distribution, and transmuted (TM) distribution [25].

To compare the competing models, the Anderson-Darling $\left(\mathrm{M}_{1}\right)$ and Cramer-von Mises $\left(\mathrm{M}_{2}\right)$ statistics are used. The model with the fewest measure values is considered sufficient for fitting the data.

Data described in $(0,1)$ can be of several types, such as percentages or proportions. Based on positive evidence $x_{1}, \ldots, x_{n}$, one can suppose that a phenomena can be schemed by a random variable $U$, with $m=\sup \left(x_{1}, \ldots, x_{n}\right)$ or any reasonable bigger number indicating the upper limit of its theoretical support. Then we may consider the random variable $X=U / m$, which is specified on $(0,1)$. In all stages, we may reconstruct the distribution of $U$ a posteriori by multiplying by $\mathrm{m}$. This problem is taken into account for the suggested data sets. The preceding technique is used for the first data set, but the remaining two data sets contain proportions-like data that is originally conveyed with values in $(0,1)$.

The reference [26] gave statistics on the number of times in months that renal dialysis patients were infected. In this data, we divide by 30 to get values between 0 and 1 . As a result, the converted data is:

$\begin{array}{llllll}0.08333333 & 0.08333333 & 0.11666667 & 0.11666667 & 0.11666667 & 0.15000000 \\ 0.18333333 & 0.21666667 & 0.21666667 & 0.25000000 & 0.25000000 & 0.25000000 \\ 0.25000000 & 0.28333333 & 0.31666667 & 0.35000000 & 0.38333333 & 0.41666667 \\ 0.41666667 & 0.45000000 & 0.48333333 & 0.48333333 & 0.71666667 & 0.71666667 \\ 0.75000000 & 0.75000000 & 0.85000000 & 0.91666667 & & \end{array}$

The second data set is made up of 48 rock samples taken from a petroleum reserve. The data are from 12 core samples taken from petroleum reservoirs and analysed using four cross-sections. Permeability was evaluated in each core sample, and each cross-section contains the following variables: total area of pores, total perimeter of pores, and shape. We examine the shape perimeter using the squared (area) variable. The information is as follows:

$\begin{array}{llllll}0.0903296 & 0.2036540 & 0.2043140 & 0.2808870 & 0.1976530 & 0.3286410 \\ 0.1486220 & 0.1623940 & 0.2627270 & 0.1794550 & 0.3266350 & 0.2300810 \\ 0.1833120 & 0.1509440 & 0.2000710 & 0.1918020 & 0.1541920 & 0.4641250 \\ 0.1170630 & 0.1481410 & 0.1448100 & 0.1330830 & 0.2760160 & 0.4204770 \\ 0.1224170 & 0.2285950 & 0.1138520 & 0.2252140 & 0.1769690 & 0.2007440 \\ 0.1670450 & 0.2316230 & 0.2910290 & 0.3412730 & 0.4387120 & 0.2626510 \\ 0.1896510 & 0.1725670 & 0.2400770 & 0.3116460 & 0.1635860 & 0.1824530 \\ 0.1641270 & 0.1534810 & 0.1618650 & 0.2760160 & 0.2538320 & 0.2004470\end{array}$

The third data set comprises 20 observations of flood data and was studied in [27]. The data are scheduled as follows:

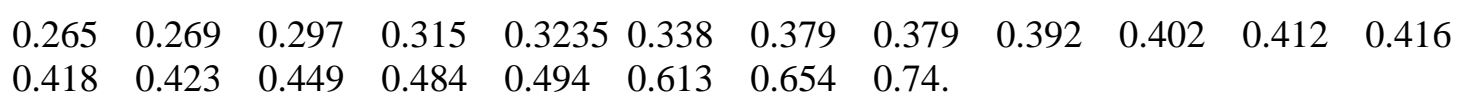




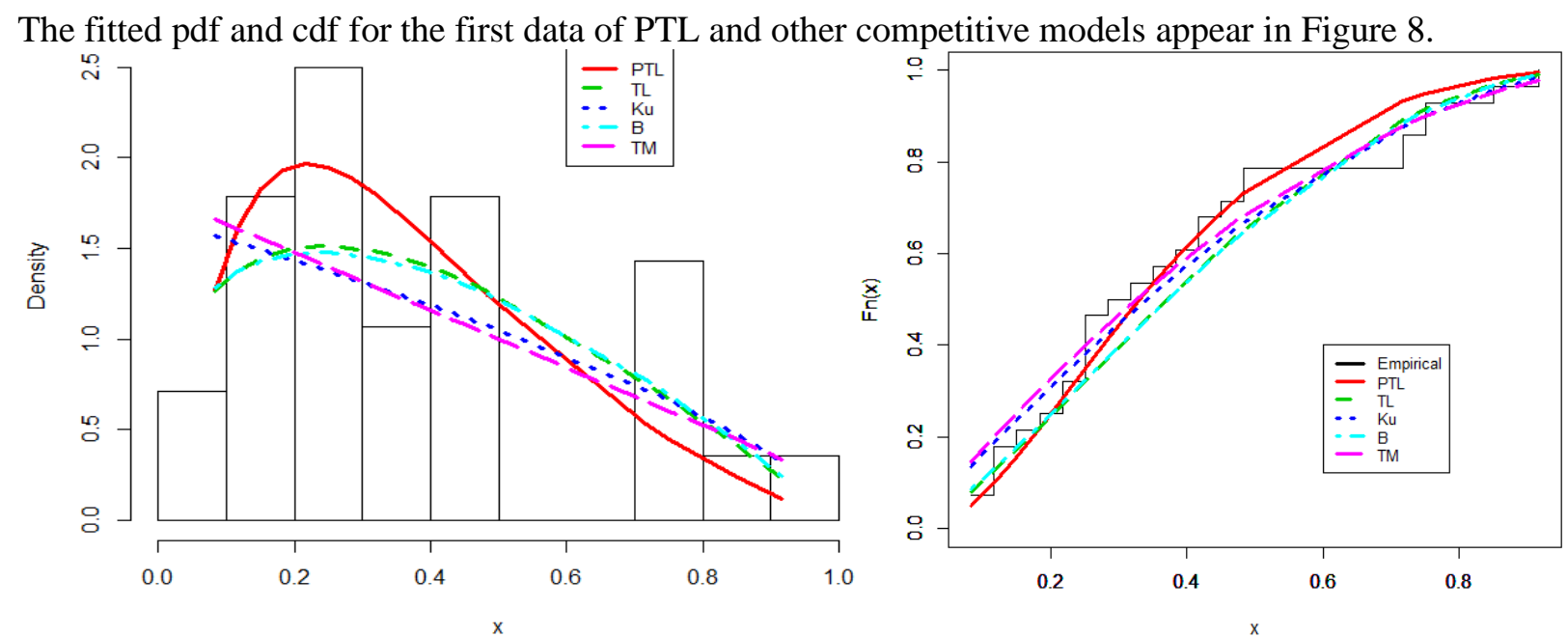

Figure 8. Fitted pdfs and cdfs of different models for the first data

Figure 8 showed that the PTL distribution is a more suitable model than the other competitive model based on the considered data.

The MLEs and their standard errors (SEs) for of the all competitive distributions are given in Table 8. Also, statistic measures $\mathrm{M}_{1}$ and $\mathrm{M}_{2}$ are mentioned in the same table.

Table 8. The MLEs, SEs $M_{1}$ and $M_{2}$ for the first data

\begin{tabular}{|ccccc|}
\hline Model & \multicolumn{2}{c|}{ MLE \& SEs } & $\mathbf{M}_{\mathbf{1}}$ & $\mathbf{M}_{\mathbf{2}}$ \\
\hline $\mathrm{PTL}(\gamma, \beta)$ & $\mathbf{3 1 . 9 4 7}(\mathbf{1 3 0 . 1 6 6})$ & $\mathbf{0 . 1 4 3}(\mathbf{0 . 3 2 3})$ & $\mathbf{0 . 4 9 0 5}$ & $\mathbf{0 . 0 7 3 8}$ \\
\hline $\mathrm{TL}(\gamma)$ & $1.3778(0.2603)$ & 0.6678 & 0.1066 \\
\hline $\mathrm{Ku}(\alpha)$ & $1.6615(0.3140)$ & 0.6897 & 0.1109 \\
\hline $\mathrm{B}(\alpha)$ & $1.3085(0.2151)$ & 0.6839 & 0.1097 \\
\hline $\mathrm{TM}(\lambda)$ & $0.7936(0.2721)$ & 0.6172 & 0.0963 \\
\hline
\end{tabular}

The likelihood ratio (LR) test statistic to test the hypotheses $\mathrm{H}_{0}: \beta=1$ versus $\mathrm{H}_{1}: \beta \neq 1$, for the first data set $\omega=4.091, \chi_{1,0.05}^{2}=3.841$, so we reject the null hypothesis.

Table 9. The MLE, LSE, WLSE, CVE, $M_{1}$ and $M_{2}$ for the second and third data sets

\begin{tabular}{|ccccccc|}
\hline & Method & Estimators of $\boldsymbol{\gamma}$ & Estimators of $\boldsymbol{\beta}$ & KS & $\mathbf{M}_{\mathbf{1}}$ & $\mathbf{M}_{\mathbf{2}}$ \\
\hline Second Data & $\mathbf{M L}$ & $\mathbf{9 2 . 1 8 8}$ & $\mathbf{0 . 0 6 7}$ & $\mathbf{0 . 2 9 7}$ & $\mathbf{1 6 . 8 1 5 1 2}$ & $\mathbf{1 . 0 6 4 1 4}$ \\
\cline { 2 - 7 } & $\mathbf{L S}$ & 0.000188 & 2464 & 0.34557 & 80.68553 & 2.531433 \\
\cline { 2 - 7 } & $\mathbf{W L S}$ & 0.00043 & 1156 & 0.351 & 77.50397 & 2.488131 \\
\cline { 2 - 7 } & $\mathbf{C V}$ & 0.0001094 & 4237 & 0.34518 & 80.74911 & 2.53225 \\
\hline Third Data & $\mathbf{M L}$ & $\mathbf{9 3 . 4 7 7}$ & $\mathbf{0 . 1 1 6}$ & $\mathbf{0 . 2 8 8}$ & $\mathbf{2 . 2 9 9 3 2}$ & $\mathbf{0 . 1 4 5 1 6}$ \\
& $\mathbf{L S}$ & 0.001199 & 694.971 & 0.331 & 15.0362 & 0.486853 \\
\cline { 2 - 7 } & WLS & 0.000321 & 2823 & 0.322 & 14.18765 & 0.47067 \\
\cline { 2 - 7 } & $\mathbf{C V}$ & 0.000154 & 5439 & 0.3298 & 15.02015 & 0.4861 \\
\hline
\end{tabular}



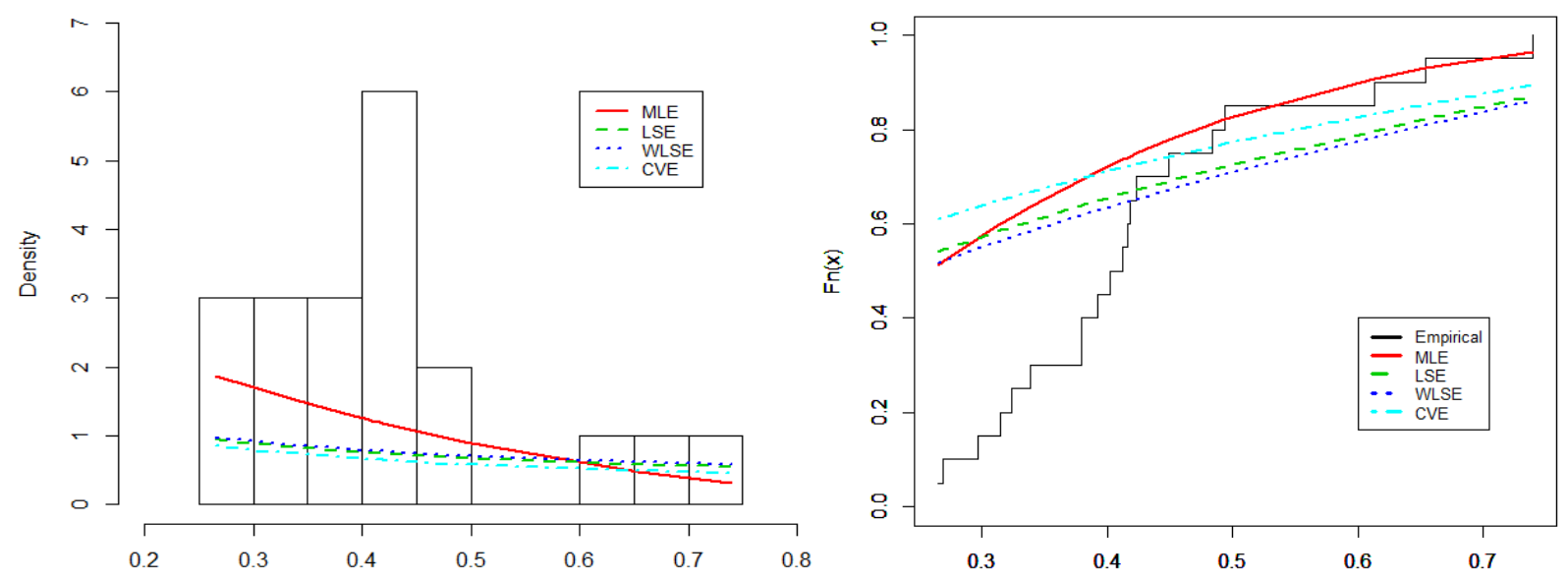

Figure 9. Fitted pdfs and cdfs of PTL model under various estimation methods for the second data
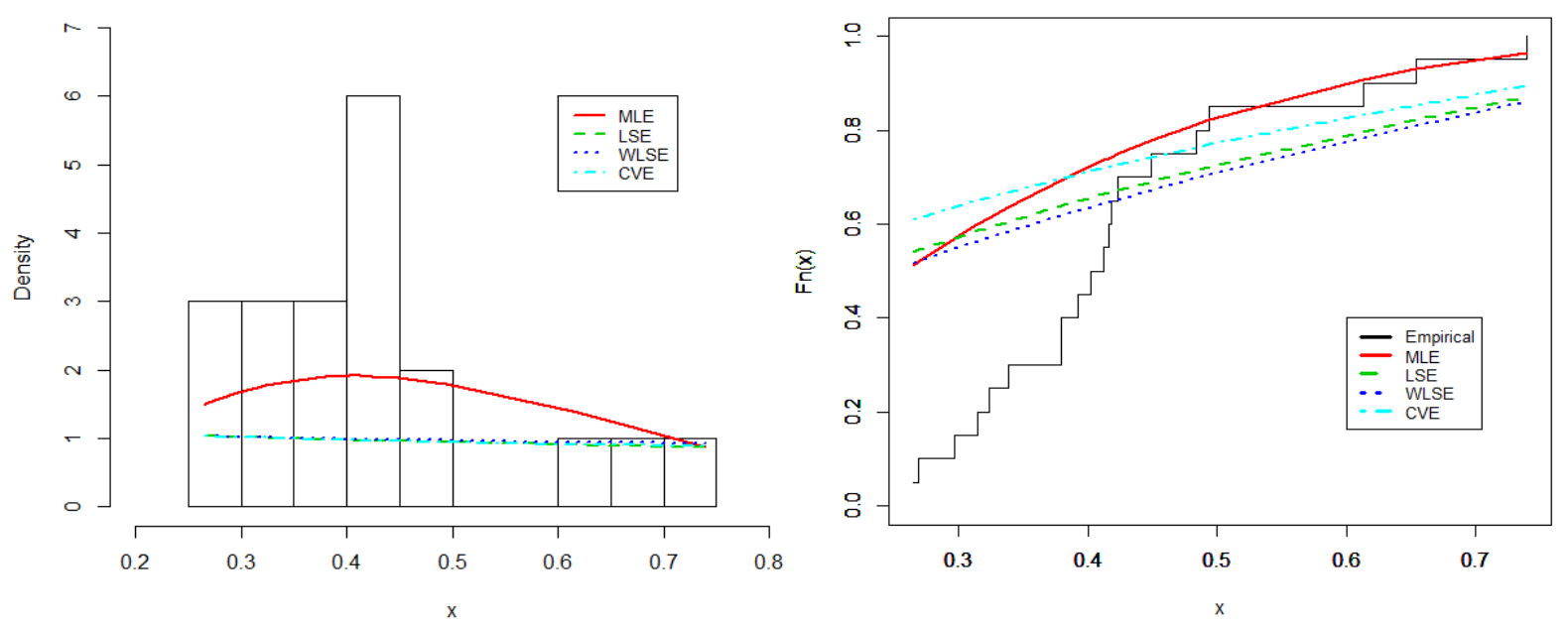

Figure 10. Fitted pdfs and cdfs of PTL model under various estimation methods for the third data

In addition, it can be confirmed from Table 8 and Figure 8 that the PTL distribution is preferable than the competitive models for the first data since it has the minimum values of $\mathbf{M}_{1}$ and $\mathbf{M}_{2}$. Further, the second and third real data are used to estimate PTL parameters from different methods as provided in Table 9 and Figures 9 and 10. We deduce that ML estimation method is the best among the others in both real data.

\section{CONCLUDING REMARKS}

We provide a novel model, the power Topp-Leone distribution. The fundamental characteristics of the PTL distribution are studied. Four distinct estimating methods are used to estimate the PTL's population parameters. To demonstrate theoretical findings, simulation and application to real-world data are explored.

\section{CONFLICTS OF INTEREST}

No conflict of interest was declared by the authors.

\section{ACKNOWLEDGEMENT}

The authors would like to thank the Editor-in-Chief and the anonymous referees for their insightful remarks, which helped to enhance the article significantly. 


\section{REFERENCES}

[1] Topp, C. W., Leone, F. C., "A family of J-shaped frequency functions", Journal of the American Statistical Association, 50(269): 209-219, (1955).

[2] Nadarajah, S., Kotz, S., "Moments of some J-shaped distributions", Journal of Applied Statistics, 30(3): 311-317, (2003).

[3] Kotz, S., Dorp, J. R. V., "Beyond Beta: Other Continuous Families of Distributions With Bounded Support And Applications", Singapore, World Scientific, (2004).

[4] Ghitany, M. E., Kotz, S., Xie, M., "On some reliability measures and their stochastic orderings for the Topp-Leone distribution”, Journal of Applied Statistics, 32(7): 715-722, (2005).

[5] Genç, A. İ., "Moments of order statistics of Topp-Leone distribution", Statistical Papers, 53(1): 117-131, (2010).

[6] Abbas, S., Taqi, S. A., Mustafa, F., Murtaza, M., Shahbaz, M. Q., "Topp-Leone inverse Weibull distribution: theory and application", European Journal of Pure and Applied Mathematics, 10(5): 1005-1022, (2017).

[7] Sudsuk, A., Bodhisuwan, W., "The Topp-Leone geometric distribution", 12th International Conference on Mathematics, Statistics, and Their Applications (ICMSA): IEEE: 108-112, (2016).

[8] Yousof, H. M., Korkmaz, M. Ç., "Topp-Leone Nadarajah-Haghighi distribution”, İstatistikçiler Dergisi: İstatistik ve Aktüerya, 10(2): 119-127, (2017).

[9] Atem, B. A., Nasiru, S., Nantomah, K., “Topp-Leone linear exponential distribution”, Stochastics and Quality Control, 33(1): 31-43, (2018).

[10] Bantan, R. A., Jamal, F., Chesneau, C., Elgarhy, M., "A new power Topp-Leone generated family of distributions with applications", Entropy, 21(12): 1177, (2019).

[11] Hassan, A. S., Elgarhy, M., Ahmad, Z., "Type II generalized Topp-Leone family of distributions: properties and applications", Journal of Data Science, 17(4): 638-659, (2019).

[12] Bantan, R. A., Jamal, F., Chesneau, C., Elgarhy, M., "Type II power Topp-Leone generated family of distributions with statistical inference and applications", Symmetry, 12(1): 1-24, (2020).

[13] Al-Marzouki, S., Jamal, F., Chesneau, C., Elgarhy, M., “Topp-Leone odd Fréchet generated family of distributions with applications to Covid-19 datasets", CMES-Computer Modeling in Engineering and Sciences, 125: 437-458, (2020).

[14] Hassan, A. S., Elgarhy, M., Ragab, R., "Statistical properties and estimation of inverted ToppLeone Distribution”, Journal of Statistics Applications \& Probability, 9(2): 319-331, (2020).

[15] Hassan, A. S., Khaleel, M. A., Nassr, S. G., "Transmuted Topp-Leone power function distribution: Theory and application”, Journal of Statistics Applications \& Probability, 10(1): 215-227, (2021).

[16] Hassan, A. S., Ismail, D. M., "Estimation of parameters of Topp-Leone inverse Lomax distribution in presence of right censored samples", Gazi University Journal of Science, 34(4), (2021). DOI: $10.35378 /$ gujs. 773645

[17] Gündüz, S., Korkmaz, M. Ç., "A new unit distribution based on the unbounded Johnson distribution rule: The unit Johnson SU distribution", Pakistan Journal of Statistics and Operation Research, 16(3): 471-490, (2020). 
[18] Korkmaz, M. Ç., "The unit generalized half normal distribution: A new bounded distribution with inference and application", University Politehnica Bucharest Scientific Bulltien Series A- Applied Mathematics and Physics, 82(2): 133-140, (2020).

[19] Korkmaz, M. Ç., "A new heavy-tailed distribution defined on the bounded interval: the logit slash distribution and its application", Journal of Applied Statistics, 47(12): 2097-2119, (2020).

[20] Hassan, A. S., Sabry, M., Elsehetry, A., "Truncated power Lomax distribution with application to flood data", Journal of Statistics Applications \& Probability, 9(2): 347-359, (2020).

[21] Korkmaz, M. Ç., Chesneau, C., "On the unit Burr-XII distribution with the quantile regression modeling and applications", Computational and Applied Mathematics, 40(1): 1-26, (2021).

[22] Shaked, M., Shanthikumar, J. G., "Stochastic Orders", Springer Series in Statistics, New York, Springer, (2007).

[23] Johnson, N. L., Kotz, S., Balakrishnan, N., Continuous Univariate Distributions, (Vol. 2), New York John Wiley \& Sons, Inc., (1995).

[24] MacDonald, P. D. M., "Comments and Queries Comment on "An estimation procedure for mixtures of distributions" by Choi and Bulgren", Journal of the Royal Statistical Society, Series B (Methodological), 33(2): 326-329, (1971).

[25] Shaw, W. T., Buckley, I. R., "The alchemy of probability distributions: beyond Gram-Charlier expansions, and a skew-kurtotic-normal distribution from a rank transmutation map", IMA Conference on Computational Finance, De Morgan House, London: arXiv:0901.0434, (2009).

[26] Klein, J. P., Moeschberger, M. L., "Survival Analysis: Techniques for Censored and Truncated Data", Second Edition, Springer-Verlag New York, Inc., (2006).

[27] Dumonceaux, R. H., Antle, C. E., "Discriminating between the log-normal and Weibull distribution", Technometrics, 15(4): 923-926, (1973). 